\title{
Wie werden die Materialkosten korrekt verrechnet?
}

\author{
Frage von Dr. med. A., Arzt für Allgemein- \\ medizin: \\ Ich mache in der Praxis regelmäßig Infusi- \\ onen als Individuelle Gesundheitsleistung. \\ Was kann ich von den anfallenden Materi- \\ alkosten korrekt verrechnen? \\ Antwort: Für diese Frage ist in der GOÄ der \\ $\S 10$ "Ersatz von Auslagen" zuständig. Hier \\ heißt es: „Neben den für die einzelnen ärzt- \\ lichen Leistungen vorgesehenen Gebühren \\ können als Auslagen nur berechnet werden \\ 1. die Kosten für diejenigen Arzneimittel, \\ Verbandmittel und sonstigen Materialien, \\ die der Patient zur weiteren Verwendung \\ behält oder die mit einer einmaligen An- \\ wendung verbraucht sind, soweit in Absatz \\ 2 nichts anderes bestimmt ist, ... Die Berech- \\ nung von Pauschalen ist nicht zulässig." \\ Der Absatz 2 ist ausschließend und gleich- \\ zeitig abschließend: „2. Nicht berechnet \\ werden können die Kosten für 1. Kleinma- \\ terialien wie Zellstoff, Mulltupfer, Schnellver- \\ bandmaterial, Verbandspray, Gewebekleb-
}

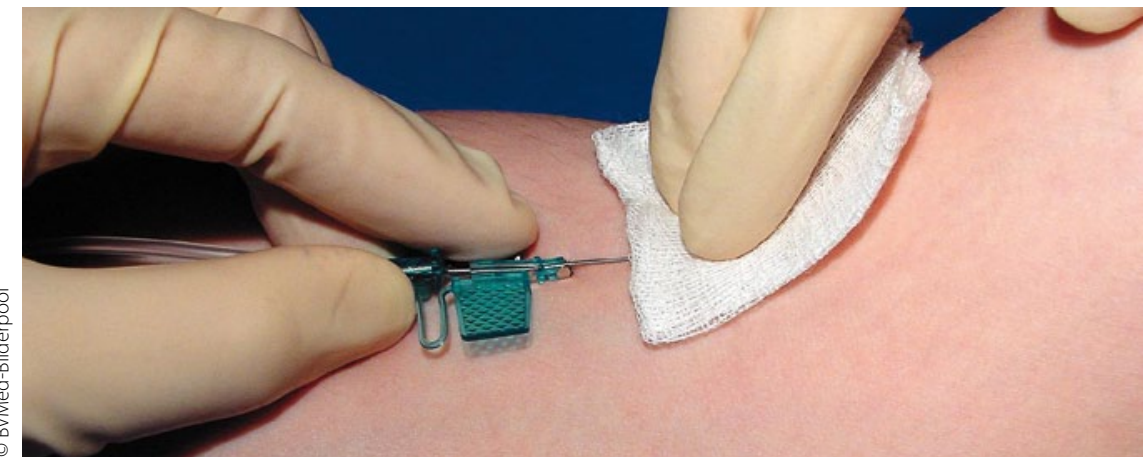

Die tatsächlichen Kosten einer Venüle dürfen in Rechnung gestellt werden.

stoff auf Histoacrylbasis, Mullkompressen, Holzspatel, Holzstäbchen, Wattestäbchen, Gummifingerlinge, ... 5. folgende Einmalartikel: Einmalspritzen, Einmalkanülen, Einmalhandschuhe, Einmalharnblasenkatheter, Einmalskalpelle, Einmalproktoskope, Einmaldarmrohre, Einmalspekula."

Somit sind bei Infusionen regelmäßig verrechenbar: die Venüle oder der Butterfly, das Infusionsbesteck, die Infusionslösung und

\section{Unfall beim 1-Euro-Job}

\section{Ist da auch die Berufsgenossen- schaft zuständig?}

Frage von Dr. med. O. S., Hausärztin: Mich suchte eine Patientin mit einer Quetschverletzung eines Fingers auf. Nach Notfallversorgung habe ich sie mittels Überweisung an einen Chirurgen weitergeleitet. Von dort erhielt ich einen D-Arzt-Bericht, obgleich die Patientin nur 1-Euro-Jobberin ist. Kann ich nachträglich einen BG-Fall daraus machen?

Antwort: Ja. Sie müssen lediglich die Daten aus dem D-Arzt-Bericht übernehmen.
Es reichen die persönlichen Daten der Patientin, der Arbeitgeber, der Unfallbetrieb, das Unfalldatum. Die Liquidation geht an die vom Unfallarzt angegebene Berufsgenossenschaft. Der Unfallarzt ist gehalten, geringfügige Verletzungen an den Überweiser/Hausarzt zur weiteren Behandlung zurückzuüberweisen. Die in diesen Fällen anfallenden Leistungen werden nach der UV-GOÄ abgerechnet. das oder die Medikamente, die mit der Infusion verabreicht werden. Berechenbar sind nur die tatsächlichen Kosten. Rabatte, Boni o. Ä. müssen in Ansatz gebracht werden. Skonti müssen nicht abgezogen werden. Es ist allerdings ein weiterer Paragraf der GOÄ zu beachten: Gemäß § 12 GOÄ sind bei der Berechnung von Auslagen nach $\S 10$ der Betrag und die Art der Auslage anzugeben: „Übersteigt der Betrag der einzelnen Auslage EUR 25,56, ist der Beleg oder ein sonstiger Nachweis beizufügen."

Da bei einer Infusion regelmäßig der Betrag von 25 EUR überschritten werden dürfte, empfiehlt es sich, auf den Namen des Patienten ein Rezept auszustellen und dies von der Lieferapotheke quittieren zu lassen. Es genügt auch eine Kopie der Rechnung der Lieferapotheke. Materialien wie Venüle und Infusionsbesteck können dann entweder als Auslage oder als Einzelverordnung berechnet werden. Auch hier sollte eine Rechnungskopie über die Bezugskosten vorgehalten werden.

Damit Sie berufsrechtlich und gegenüber dem Patienten auf der sicheren Seite sind, sollten diese Kosten in einem „Behandlungsvertrag", wie er für jede IGeL notwendig ist, vorher vom Patienten als zur Kenntnis genommen bestätigt werden. 